\title{
La Real Sociedad Patriótica de Amigos del País del reino de Córdoba (1779-1810)
}

\author{
Maria del Carmen Gil Romero *
}

Con el advenimiento al trono de Carlos III en 1759 la renovación parece llegar a toda España. En el reinado de este monarca penetran en el pais nuevas ideas, y una nueva mentalidad se instaura en el Gobierno. Se tiende hacia un predominio de la razón y las ciencias útiles. La renovación se centra en la racionalización de la Sociedad Estamental.

Esta nueva mentalidad es el despotismo ilustrado, que fue resumido por Palacio Atard ${ }^{1}$ en cuatro grandes puntos:

1. Político-religioso: intenso desarrollo del regalismo.

2. Politico-administrativo: centralización.

3. Económico-social: amplias y variadas medidas que constituyen un vasto programa de reformas.

4. Cultural: procurar elevar el nivel cultural y fijar la atención en aspectos científicos hasta entonces no cultivados.

* Profesora-tutora del Centro Asociado de Córdoba.

' V. PALACIO ATARD: "El despotismo ilustrado español», en Historia de España, de Arbor, pp. 360-361. 
Los representantes de esta nueva mentalidad, los ilustrados, comenzaron reuniéndose y formando tertulias y academias locales en las ciudades más importantes, por lo que vemos multiplicarse durante todo el siglo las discusiones y las agrupaciones literarias y científicas a lo largo de todo el territorio español.

De esta manera surge la primera Sociedad Económica. En Azcoitia todas las noches se reúnen los caballeros y sacerdotes del lugar dando origen a la Sociedad Vascongada de Amigos del País. Estas Sociedades se extendieron por toda España a partir del último tercio del siglo XVIII y la época de máxima formación se sitúa entre 1765 y 1786 aproximadamente. Surgen las Sociedades Económicas como un reflejo de la llustración y son el instrumento que más contribuye a defender esta ideología y al mismo tiempo a fomentar y promover los estudios de Economía Política y el desarrollo local. Desde la fundación de la Sociedad Vascongada en 1765, hasta la edición en 1774 del Discurso sobre el fomento de la Industria Popular de Campomanes, sólo se organizan las sociedades de Baeza y Tudela. Pero a partir de 1774, con la difusión del discurso y de la Real Cédula van surgiendo en cualquier ciudad o villa que tenga un núcleo ilustrado estas sociedades. Desde un principio fueron protegidas por el Gobierno pero su fundación fue dejada a la iniciativa particular de grupos de personas.

Marcelo Bitar Letayf piensa que las sociedades eran entidades populares que tenian diferentes objetivos: impulso de la agricultura y la ganadería, en colaboración directa a veces con el labrador, a quien facilitaban gratuitamente semillas; prestaban también su apoyo a cualquier mejoramiento industrial, a través muchas veces de clases y enseñanzas prácticas ${ }^{2}$.

La dispersión geográfica de las Sociedades nos muestra la existencia de un núcleo importante en Andalucia. Según consta en los Expedientes de fundación que se conservan en el Archivo Histórico Nacional en la sección de Consejos Suprimidos, la primera que nació fue la de Baeza con fecha de solicitud del 8 de mayo de 1774 y su aprobación por el Consejo fue realizada el 31 de mayo de 1774, siguiendo una lista que consta de 31 sociedades.

${ }^{2}$ M. Bitar Letayf: Economistas españoles del siglo xvil, Ediciones Culturales Hispanas. Madrid 1968, p. 17. 
Para conocer las sociedades que se crearon en el Reino de Córdoba contamos con los estudios realizados por Gonzalo Anes ${ }^{3}$ que nombra a ocho sociedades y son las de Priego, Cabra, Montilla, Bujalance, Córdoba, Lucena, Aguilar y Baena. En segundo lugar contamos con el libro Guía del Ministerio de la Gobiernación del Reino para el presente año $1836^{4}$, en el cual se citan junto con las anteriores los nombres de cinco sociedades más que son las de Castro del Rio, Fuente Obejuna, Montoro, Pozoblanco y La Rambla, llegando el número de sociedades por lo tanto a 13 en 1836.

Debido al amplio número de sociedades existentes y a los pocos estudios realizados sobre el tema, apuntaré algunos datos de varias de éstas y me detendré en la Real Sociedad Patriótica de Córdoba, dando unas pinceladas de su historia desde su fundación hasta que desaparece en 1921, y profundizando en la actividad desarrollada en un periodo muy concreto, el que va de 1779 a 1811.

REAL SOCIEDAD LABORIOSA DE LA M. N. Y M. L. CIUDAD DE LUCENA $^{5}$

En 1779 existe en Lucena un grupo de ilustrados que a raíz del discurso de Campomanes deciden formar la Real Sociedad Laboriosa de la M. N. y M. L. ciudad de Lucena. Este movimiento ilustrado fue muy fuerte y muchos de sus miembros fueron fundadores de otras sociedades al igual que miembros honorables.

Los estatutos fueron redactados por una comisión de tres socios, a imagen de los que poseian las Sociedades de Madrid y de Sevilla. Estos estatutos se aprobaron el 5 de abril de 1780 e intentados renovar en el siglo XIX.

${ }^{3}$ Gonzalo ANES: Economía e Ilustración en la España del siglo xvill. Ed. Ariel, Barcelona 1981, pp. 26-29.

4 Guia del Ministerio de la Gobernación del Reino para el presente año 1863, Madrid, Imprenta Real, 1836.

5 J. A. Abras Santiago: "La Real Sociedad Laboriosa de la M. N. y M. L. Ciudad de Lucena. Andalucia Moderna (S. xvII)". Actas del / Congreso de Historia de Andalucía, dic. 1976, tomo I, pp. 3-12. 


\section{REAL SOCIEDAD ECONÓMICA DE AMIGOS DEL PAÍS DE CABRA ${ }^{6}$}

En agosto de 1779 , todas las fuerzas vivas de la ciudad acuerdan constituir la Sociedad Económica de Amigos del País de Cabra. La reunión fue presidida por D. Miguel Jiménez Navarro, Caballero de la Orden de $D$. Juan e Intendente General de la ciudad de Córdoba y su provincia; también asisten los personajes importantes de la villa y todos los vecinos convocados a Cabildo Abierto.

Sus estatutos debían de ajustarse a los de Sevilla y su finalidad principal fue la de ejercitar la industria, habilitando las fábricas y artefactos adecuados para facilitar el logro de la prosperidad de los naturales y vecinos.

La aprobación de los estatutos de la Sociedad pasaron por grandes vicisitudes, conociéndose dos fechas de solicitud: la del 21 de agosto de 1779 y la del 13 de junio de 1788, pero no fueron definitivamente aprobados y bajo la protección real hasta el 10 de octubre de 1790 .

\section{REAL SOCIEDAD ECONÓMICA DE PRIEGO DE CÓRDOBA}

Aprobada su fundación en 1779 , fue su primer director D. Esteban Lorenzo de Mendoza, abad de Alcalá la Real. No se tiene noticia de que llegara a formar estatutos.

\section{REAL SOCIEDAD ECONÓMICA DE AMIGOS DEL PAÍS DE POZOBLANCO $^{7}$}

Las fuentes documentales con las que contamos para el estudio de esta Sociedad Económica son muy escasas: documentos privados y varias referencias en archivos. Debido a tan inexistente información, en un principio se pensó que la Sociedad que existió en Pozoblanco sería una

6 J. Garcia Garcia: “La Real Sociedad Económica de Amigos del Pais de Cabra (Córdoba). Andalucia Moderna (s. Xviti)". Actas del I Congreso de Historia de Andalucía, dic. 1976, tomo I.

7 A. Muñoz Calero: Sociedades Económicas de Amigos del País, Publicaciones Cajasur, 1985, pp. 5-7. 
delegación de la Sociedad Económica Cordobesa. Pero ello no es cierto puesto que si nos basamos en las noticias que sobre cierto corregidor llamado D. Dionisio Catalán existen, comprobaremos que en 1807 y 1808, es decir, a comienzos del siglo XIX, en Pozoblanco funcionaba una Sociedad Económica. Esta idea es confirmada en la Guía del Ministerio de la Gobiernación del Reino para el presente año de 1836, donde se recogen los nombres de sus directivos.

Las actividades que desarrolló la sociedad de Pozoblanco en la comarca de los Pedroches debió de ser importante por el propio carácter de la zona; podemos encontrar en ella industrias de manufacturas y agricolas, comercio e incluso problemas de repartición de la tierra. Los consejos de la Sociedad sirvieron para impulsar las reformas y las ideas de la ilustración.

\section{REAL SOCIEDAD ECONÓMICA DE AGUILAR DE LA FRONTERA}

Fue fundada el 19 de julio de 1786 por iniciativa del duque de Medinaceli. Se utilizó como lema el de "Favorece Instruyendo". Se preocupó la Sociedad del Establecimiento de escuelas de aritmética y primeras letras. Proyectó la fundación de "Casas de misericordia" y fábrica de telas y, especialmente, de la industria de la lana. Se extinguió hacia 1815.

\section{REAL SOCIEDAD ECONÓMICA DE BAENA}

Creada por D. Miguel Cañete, procurador síndico en 1787, prestó especial atención al desarrollo de la agricultura y la industria de lienzos. Parece que dejó de existir hacia fines del siglo XVIII. Fue su primer director el presbítero D. Eugenio Caballero.

\section{REAL SOCIEDAD ECONÓMICA DE MONTILLA}

Los únicos datos que poseemos son la fecha de su fundación en 1779 y que sus estatutos no llegaron a redactarse. 


\section{REAL SOCIEDAD ECONÓMICA DE BUJALANCE}

D. Teodoro Espinosa de los Monteros fue su fundador, promotor y primer director en 1780. Prestó especial atención a la creación de fábricas de papel, sombreros y curtidos. Debió de extinguirse a principios del siglo XIX.

\section{REAL SOCIEDAD PATRIÓTICA DE CÓRDOBA}

La Real Sociedad Patriótica de Córdoba debe su origen a la preocupación de los señores D. Gregorio Pérez Pavía, presbítero, D. Bartolomé Basabru, D. Blas Antonio de Cadenas y D. Diego Bonrostro y Carasquilla, que reunidos el 10 de marzo de 1779: viendo el espectáculo natural y común de la miseria, a que en este siglo están reducidos las más de las personas pobres, muchachos y jóvenes de ambos sexos mendicando su alimento, desnudos y en abandono de las costumbres cristianas, así como la decadencia que desde hace tiempo viene sufriendo esta ciudad. Convencen a D. Pablo Tomás de Vidaurreta, D. Nicolás de Fuentes, D. Miguel Barcia, D. Andrés de Baena, D. Sebastián Alfonso de Lea y $\mathrm{D}$. Bartolomé Baquerizo, para que se hagan suyas estas causas ya que no se había establecido en la ciudad de Córdoba Sociedad Económica de Amigos del país como en otros lugares, con personas llenas de humanidad que han establecido industrias populares, fomentando las artes y las manufacturas, procurando hacer útiles al Estado todas las personas, habilitándolas de enseñanza e instrumentos, y especialmente de moral cristiana. Se adhirieron voluntariamente a este asunto los señores Baylio, Marqués de la Vega, D. Pedro de la Cerca, Marqués de Guadlacar, D. Damián de Castro y Garcia y D. Eugenio Alfaro ${ }^{8}$.

Reunidos en 6 de junio de 1779, los señores anteriormente nombrados, deciden fundar una Sociedad de Amigos del País. Piensan estos primeros socios que todo el mal se encuentra en la falta de educación, enseñanza y aplicación en la infancia y para ello fomentan el Colegio de Niñas Pobres, por lo que piden en esta fecha la protección del Real Consejo para ambas instituciones.

${ }^{8}$ Libro primero de acuerdos de la Sociedad Patriótica de Córdoba. 
La vida de la Sociedad Económica Cordobesa puede dividirse en varias etapas siguiendo algunas de gran eficacia y obras de gran nulidad. De 1779 a 1810, centra su actividad y su preocupación en una sola tarea: el Colegio de Educandas, como podemos comprobar en las actas de este período. La Sociedad era muy poco extensa, como sus propios socios indican "reinaba cierto espíritu que reducía la corporación a una oligarquía" ". A partir de 1802 con la entrada de nuevos socios se renuevan las ideas sobre los fines que debian regir en ella, esta nueva mentalidad queda reflejada en el encargo que recibió D. Manuel María de Arjona en 1803 de realizar las constituciones de la Sociedad y el Colegio.

En 1810 con la admisión de nuevos socios la Sociedad adquiere una mayor eficacia creando cátedras como la de Historia, Economía Política, Químicas, Nobles Artes, Legislación Universal, Agricultura y al mismo tiempo se divide en secciones como la de población, agricultura, comercio, etc... Este nuevo impulso le llevará a participar en la Junta de Socorro Público o de Beneficiencia y en la creación de juntas parroquiales.

A partir de esta fecha no tenemos noticia de las acciones de la Economía, sí aparecen en documentos posteriores de la misma referencia a una expropiación de sus propiedades por parte del Gobierno, por lo que podemos suponer que sus actividades quedaron paralizadas hasta el 2 de marzo de 1841, según las referencias tomadas del historiador Ramírez de las Casas-Deza. La labor realizada en esta etapa fue precaria debido sobre todo a la falta de medios económicos ${ }^{10}$.

Un nuevo resurgir tuvo lugar con la monarquía de Alfonso XII. Comenzaron a reeditar el Boletín mensual, se mantuvo una nutrida y fluida correspondencia con otras Sociedades Económicas y con otras instituciones de carácter cultural y científico, se celebraron certámenes literarios, etcétera. Pero sobre todo debemos destacar cuatro realizaciones en el plano de la enseñanza: la Escuela de Artes y Oficios, la Escuela Nocturna, la Escuela de Comercio y la Academia gratuita de Idiomas.

Conocida, aunque muy someramente, la vida de una Sociedad Económica de provincias como es la Cordobesa, pasaremos al estudio más

9 Noticia Histórica de la Real Sociedad Patriótica de Amigos del País de Cordoba (1779-1816). Imprenta del Diario Córdoba, Córdoba 1816.

10 Ramírez de las CaSAS-Deza, Indicador..., p. 259, vid también Memorias...., Córdoba en el s. XIX, p. 105. 
pormenorizado de un período muy concreto, el que discurrió entre los años de 1779 a 1811, fechas a mi entender claves. La primera, como puede comprobarse a simple vista - fecha de fundación-y la segunda un poco más complicada, puesto que se trata de una apreciación muy personal una vez analizada el acta del 11 de junio de 1811, de la cual se desprende un intento de la Sociedad Económica de cumplir sus normas, puesto que se revisan todas las actas anteriores, tratando de promover una imagen de seriedad y buen funcionamiento tal y como corresponde a una institución de su índole.

Uno de los documentos que mejor nos informan sobre la Económica Cordobesa, las primeras constituciones, no se conocen. Sí se conservan constituciones posteriores, como la de 1875 y los reglamentos de funcionamiento interno de 1875, 1898 y 1920.

Como queda reflejado en junta de 13 de junio de 1779, los señores anteriormente nombrados encargaron al señor Baylío que pidiese licencia al Magistrado para el establecimiento de la Sociedad Económica de Amigos del País y del Colegio de Educandas. Posteriormente llegó un decreto del Real y Supremo Consejo de Castilla -14 de julio de 1779 por el cual se mandaban formar las reglas de la Sociedad y de la Casa de Educandas. Para la redacción de estas reglas la Sociedad nombra en comisión al señor D. Manuel Baquerizo y D. Blas Antonio de Codes, pero los trabajos fueron muy lentos y estas constituciones nunca vieron la luz.

En 1810 se plantea la Sociedad la necesidad de poseer tanto ella como el Colegio unas constituciones y se comisiona a D. Manuel María de Arjona para la redacción de ésta. Los pasos que han de recorrer las constituciones hasta su presentación y aprobación en la junta del 3 de junio de 1806 son múltiples.

En un primer momento las constituciones tuvieron que ser remitidas a la Cancillería de Granada, la cual informa a través del corregidor la necesidad de que la Sociedad nombre un procurador para su defensa ante el tribunal. El informe por fin remitido fue favorable, como también lo fue el informe del Fiscal de Su Majestad, por lo que las constituciones fueron por último aprobadas por el rey.

Para acercarnos un poco al conocimiento de estas constituciones tenemos que basarnos en las referencias que sobre ella aparecen en las actas de la juntas celebradas en este período. Sabemos que los capítulos $3 .^{\circ}, 4 .^{\circ}, 5 .^{\circ}, 6 .^{\circ}, 7 .^{\circ}, 8 .^{\circ}, 9 .^{\circ}, 10 .^{\circ}, 11 .^{\circ}$ están dedicados al tema de las 
elecciones. Para la celebración de la elección se formaba una junta de cinco miembros que posiblemente presidiría la junta de elecciones. A continuación se repartía a cada socio una lista con todos los socios, los oficios y las diputaciones. Era elegido para estos cargos el socio que más votos obtuviera.

Los cargos a elegir son: Director, Vicedirector, dos Secretarios, tres Calveros, tres Diputados de Educación, tres Diputados de Literatura, dos Diputados de Fábrica y dos Administradores del Lagar. Los primeros nombramientos se celebran el 29 de abril de 1781 y es elegido director el señor Baylío, Marqués de la Vega y director en funciones por ausencia de éste a D. Manuel Baquerizo. Se celebraron elecciones generales el 15 de noviembre de 1803 , el 22 y 29 de agosto de 1804 , el 28 de junio de 1805 , por to que podemos suponer que estas elecciones se debian de celebrar anualmente.

Estos cargos componen el plan orgánico de la Sociedad, el cual se ampliará el 4 de septiembre de 1810 con la creación de secciones que fueron: población, agricultura, artes y oficios, comercio, navegación y recursos. Las secciones cuentan con un reglamento aprobado el 17 y 18 de septiembre de 1810, y que consta de 21 puntos. Estas secciones estaban compuestas por un presidente, un secretario y diferente número de socios. El presidente será nombrado por la junta, el secretario por el presidente y los socios por el director de la Sociedad.

Una reestructuración del plan orgánico de la Sociedad se produce en 1810 quedando ésta dividida en una parte general compuesta por el director, el videdirector, el censor y los dos secretarios, y otra parte particular que, a su vez, se subdivide en interior, donde se situarian las diputaciones y exterior, donde se daría cabida a las secciones.

El capítulo 13 corresponde a la celebración de las juntas a las cuales hacemos tantas referencias. Las juntas -órgano decisivo de la Sociedad- se celebraban semanalmente. En 1803 se acuerda que éstas tuviesen lugar los viernes y en 1810 son traspasadas a los martes. Estas juntas pueden ser ordinarias, extraordinarias y secretas. Se necesitaba un número mínimo de socios que según la constitución es de nueve para que los acuerdos que se tomasen fuesen válidos, pero en junta del 4 de marzo de 1805, debido al escaso número de socios, que redujo el quorum a siete miembros. Posteriormente en la revisión de las actas que realizó la propia Económica se declaró nulo este último acuerdo. 
En relación también con las juntas nos encontramos el capítulo 5 , en el cual se dice que a falta del director y vicedirector la junta será presidida por el socio más antiguo. Este capítulo será anulado en 1810 y se dice que a partir de ahora serán suplidos por los presidentes de secciones y por el siguiente orden: literatura, población, agricultura, comercio, navegación y recursos; y si alguno es censor o secretario pasará el turno al siguiente.

Otros capítulos de los que conocemos su temática son el 9 y 10 que prescriben todo lo relativo a las cuentas, también existía un capítulo dedicado a la asociación de señoras, pero que no fue puesto en práctica y permaneció olvidado hasta 1811.

Por último, decir que para la reforma de las constituciones o reglamentos internos necesitaban tomarse tres acuerdos. Estos acuerdos no aparecen especificados en el acta, a excepción del tercero que dice que lo reformado tiene que aprobarse por unanimidad.

Desde su fundación unas de las máximas preocupaciones de la Sociedad fue la educación. Esta preocupación queda reflejada en la creación del 1 de abril de 1871 de la Casa-Colegio de Educandas, que sería administrado por la Económica. En él se acogerían a 12 niñas entre los seis y nueve años de edad, cuyos requisitos son el de ser huérfana, pobre, que sean cristianas viejas y limpias, junto con una madre-maestra. Se le enseñaría doctrina cristiana, a coser, hilar, hacer media, tejer y todas las haciendas y primores mujeriles. Dentro de esta institución se fundaron en abril de 1781 una Amiga Pública de costura y labor, y en la cual con el transcurrir del tiempo se enseñó junto con la doctrina cristiana a leer y escribir; posteriormente en noviembre de 1784 se acuerda admitir pupilas de corta edad, cuyos padres pagarian el vestido, el uniforme y una cantidad anual que en un primer momento fue de 50 ducados y cuatro fanegas de trigo. La edad de las pupilas oscilaría entre ocho y diez años y se mantendrian en el colegio hasta la edad de 20 años.

La realización de sus constituciones y reglamento interno corren paralelos a los de la Sociedad. En junta celebrada el 4 de marzo de 1806 se presentó un informe de D. Manuel María de Arjona, en el que comunica que las constituciones han sido aprobadas. Estas no se conocen por lo que no podemos hacer un estudio muy detallado del gobierno del colegio, pero si conocemos un informe de dicho señor del 2 de diciembre de 1803 sobre el estatuto y funcionamiento del colegio durante ese año. 
Según el informe el personal que lo componía era: la directora, las maestras de las educandas, las maestras de la amiga pública y las ayudantes. Junto a ellas contamos, como es natural, con las niñas que podían ser educandas, pupilas y de la amiga pública; además de la criada y el portero.

Las tareas diarias de las niñas se repartian entre las clases de doctrina cristiana, escritura, lectura, hilar a la tornilla, bordar y en ayudar a las tareas de la casa. No estaban las niñas repartidas en clases sino que todas las maestras enseñan lo que saben en las horas desocupadas.

Los recursos con los que cuenta el colegio para su mantenimiento son muy escasos. Tenemos que diferenciar dos clases de recursos, aquellos que provienen de las limosnas y subvenciones del Estado y los que le renta el lagar de Pino Gordo de su propiedad y la ayuda de la fábrica.

Entre los primeros se encuentran 15.000 reales anuales otorgados por Su Majestad el 18 de diciembre de 1799 sobre el Fondo Pío de Beneficencia de la diócesis. Estos 15.000 reales eran el sustento más importante de la Casa de Educandas y debido a su pago irregular condicionó peligrosamente su existencia. También debemos mencionar las cuotas que en determinados momentos pagaban voluntariamente algunos socios, como la cuota de 120 reales pagada en 1871 .

Entre las segundas contamos con el lagar de Pino Gordo de Valparaiso que fue donado al colegio por el socio D. Nicolás Moyano y de Armentía a su muerte, con la condición de que no fuera vendido por la Sociedad. El lagar fue valorado en 200 ducados anuales y constituyó en la mayoría de las ocasiones un problema para ésta. Una de las soluciones dadas fue su arrendamiento que se llevó a cabo a partir de 1806.

La fábrica se establecía gracias a la limosna concedida por el Comisario General de Cruzada, la cual ascendió a 8.000 reales. A raíz de ello se comienza a estudiar por parte de los socios asignados, el establecimiento de una fábrica de lino y otra de lana. Con estas creaciones se pretendía ayudar a las personas económicamente menos pudientes y a la propia Casa de Educandas mediante el trabajo realizado en ella por las niñas del colegio.

Como hemos dicho, al mismo tiempo que se aprobaron las constituciones de la Sociedad se aprobaron unas para el gobierno del Colegio, 
pero éstas no fueron utilizadas. Por ello en 1803 se vio la necesidad de un reglamento para las maestras y las niñas, y un año más tarde se pide a D. Manuel María de Arjona que se adjunte un capítulo dedicado a los exámenes, acordándose lo siguiente:

1. Los exámenes versarán sobre las ramas que la Sociedad mande.

2. Se realizarán tres exámenes por enero, mayo y septiembre.

3. Para ser despedida una alumna tendrá que tener tres suspensos seguidos o seis interpolados.

4. Los exámenes serán realizados por la diputación de educación y las maestras que nombre la Sociedad.

5. Se superarán éstos si son aprobados por seis votos, si no pasará a junta secreta.

6. Se tendrá cuidado que los defectos no sean de enseñanza de la maestra.

Las actas de este periodo - 1779 a 1810 - hacen constantemente referencia a los problemas que sufre el colegio, a las niñas que son admitidas, a la celebración de exámenes a la contratación de maestras y maestros, a los recursos, etc..., pero a partir del 13 de octubre esta preocupación hacia el colegio se diluye y se comienza a trabajar y a ampliar las miras en otros asuntos.

En agosto de 1810 a raíz de la admisión de nuevos socios parece que un nuevo espíritu invade la Sociedad. Junto a las antiguas diputaciones aparecen las secciones en donde los trabajos y memoriales se multiplican. Estos trabajos realizados con el espíritu de la llustración hacen que la Económica se amplíe en número y en fama tanto en la ciudad como en todo el reino, consultándosele temas de tan variada temática como la higiene, los cementerios, la apertura de amigas públicas en Córdoba, etc...

Junto al establecimiento de las secciones se crean una serie de cátedras. En septiembre de 1810 se crean las de matemáticas y Nobles 
Artes que se encuentran en el Colegio de la Asunción. La Cátedra de Historia creada por el señor Arjona celebrará sus secciones tanto en el Colegio de la Asunción como en la sede de la Económica.

Uno de los problemas más importantes con los que se tuvo que enfrentar la Sociedad fue el económico. Esta no contaba con fondos suficientes para el establecimiento de algunas cátedras como las de química y agricultura debido a la necesidad de un laboratorio y terrenos para hacer experimentos y trabajos prácticos. El problema se solucionó con la ayuda prestada por el Prefecto de la ciudad.

Entre los temas más tratados en estas secciones destacan en la de Agricultura: la siembra de la patata, las leyes extraordinarias y ordinaria para fomentar la cosecha de 1810, formación de viveros, fábricas de azúcar de uvas, de remolacha, de algodón, utilización de las sustancias de los huesos para alimentación. En la de Ciencias Naturales y Exactas se presentaron memoriales como "La bomba para apagar incendios" o los memoriales de D. Rafael Benítez sobre el pararrayos y su instalación en Córdoba. El memorial de D. Pedro de Cubas en el cual propone un plan de vacunación para los pobres o como el del Sr. Entemena que propuso averiguar el origen y progreso del arte de tenedores en esta ciudad y el modelo de restablecerlo.

En un apartado tan importante como es la beneficencia, la actividad de la Sociedad Económica o Patriótica se centra en la creación de instituciones como la Asociación del Buen Pastor establecida por ésta a favor de los presos, su participación en la Junta de Socorro Público, destacó también por sus ayudas al hospicio y por la lucha por la implantación de la "sopa de pobres".

Adquirieron también mucha importancia para la Sociedad el establecimiento de otras Económicas en los pueblos de la prefectura y subprefectura donde éstas no existiesen y donde existiesen mantener una relación más directa. El 23 de octubre de 1810 se leyeron copias de los oficios remitidos a las sociedades de Écija, Lucena, Bujalance y Aguilar, la cual constató el 6 de noviembre de 1810.

Se pretendieron fundar otras instituciones como una Academia Médica, cuyos estatutos fueron realizados y mandados para su aprobación por el rey; también se crea la Academia de Bellas Letras que quedó fundada el 11 de noviembre de 1810. El establecimiento de un Liceo, la apertura de una Academia de Maestras de Primeras Letras cuyos esta- 
tutos se presentan en abril de 1811, la creación de una Casa de Moneda, etcétera.

Para terminar tratar muy someramente dos apartados: el dedicado a los socios y el de los recursos de la Sociedad.

En el campo económico la Sociedad subsistía con las limosnas o cuotas pagadas por sus socios, con el dinero que daban las autoridades como el comisario general, el obispo, el prefecto y las que obtenían del lagar de la fábrica.

Dentro de los socios se distinguen los de primera fundación y los socios honorarios, y dentro de estos los honorarios los que pagan cuotas o no pagan. Las clases sociales en las que se encuadran son muy variadas aunque con predominio del clero. Así encontramos también nobles como el Marqués de la Vega y el Marqués de Guadalcázar, comerciantes como D. Nicolás de Fuentes, cargos administrativos, municipales, etcétera. 